\title{
Myco-toxins Monitoring Device and their Management Strategies through Detoxifying Agents in Feed
}

\author{
Shrvan Kumar ${ }^{1 *}$, Asha Sinha1, Shweta Meshram², Mukesh Kumar Singh ${ }^{3}$, \\ Vimla Singh ${ }^{4}$ and K.S. Hooda ${ }^{4}$
}

\author{
${ }^{1}$ Mycology and Plant Pathology, IAS, Banaras Hindu University, Varanasi (U.P.) - 221005 \\ ${ }^{2}$ Divison of Plant Pathology, IARI, New Delhi-110012, India \\ ${ }^{3}$ Genetics and Plant Breeding, IAS, Banaras Hindu University, Varanasi (U.P.) - 221005 \\ ${ }^{4}$ Indian Institute of Maize Research, IARI Campus, New Delhi-110012, India \\ *Corresponding author
}

\section{Key words \\ Mycotoxin, \\ Biosensors, \\ Detoxifying agents, \\ Adsorbing agents, \\ Bio-transforming \\ agents}

\section{Article Info}

\section{Accepted:}

26 December 2017

Available Online:

10 January 2018

\section{A B S T R A C T}

The naturally toxigenic fungal flora, existing in conjunction with food production is most dominated genera namely, Aspergillus, Fusarium and Penicillium and to lesser extent the Alternaria, Claviceps and Stachybotrys. More than 300 mycotoxins are known to exist in nature. The economically important species of fungi producing significant mycotoxins as: aflatoxins (AFs), citrinin (CIT), cyclopiazonic acid (CPA), fumonisins (FBs), moniliformin (MON), ochratoxin A (OTA), deoxynivalenol (DON), nivalenol (NIV), T-2 toxin (T-2), patulin (PAT) and zearalenone (ZEA). Several impacts on consumers, such as loss of human and animal lives; health care and veterinary care costs; contaminated foods and feeds disposal costs; and investment in research and management of the mycotoxin problem. Myco-toxins are able to induce powerful and diverse biological effects. Diverse actions of myco-toxins have been characterized on animals and humans to include cytotoxic, carcinogenic, immunosuppressive, nephrotoxic, neurotoxic, mutagenic and oestrogenic effects. Pre and post-harvest management strategies are most important for management of toxicogenic fungi in food materials. Mycotoxin-detoxifying agents for reduction of the contamination of feed by mycotoxins that substances can suppress or reduce the absorption, promote the excretion of mycotoxins or modify their mode of action. These feed additives transform mycotoxins into less toxic metabolites either by reducing their bioavailability or by degrading them. Therefore, we can define at least two main categories; including first various mycotoxin adsorbing agents and second biotransforming agents leads to degradation of mycotoxins into non-toxic metabolites. And also advanced detection techniques are help in ultra-trace amount of myco-toxin in food products as like; bio-sensors (1) electrochemical biosensors, 2) optical biosensors 3)electronic biosensors, 4)piezoelectric biosensors, 5) gravimetric biosensors, 6) pyroelectric biosensors. 


\section{Introduction}

Mycotoxins have a wide range of chemical structures and their different physiological activities range from neutral to toxic potentials (Richard, 2007; Njobeh et al., 2010b). Approximately 300-400 mycotoxins are known to exist in nature (Huff et al., 1992) in Table 3. St Anthony's fire was one of the first well-known recognized disease caused by mycotoxins in Europe. This was ergot alkaloids produced by the mold Claviceps purpurea in rye (Bove, 1970). In African continent, a cumulative effect between mycotoxin and some important diseases such as tuberculosis, malaria, kwashiorkor and AIDS/ HIV have been suggested (Turner et al., 2003; Gong et al., 2003, 2004). Nowadays, mycotoxins are recognized as causal factors for primary liver cancer, ergotism and alimentary toxic aleukia (Shephard et al., 2007). Most storage fungi grow at $a_{w}$ below 0.75 generally, the required $\mathrm{a}_{\mathrm{W}}$ for fungal growth is between 0.61 and 0.91 (Robert and Raymond, 1994). Leland C. Clark introduced the principle of the first enzyme electrode with immobilized glucose oxidase at the New York Academy of Sciences Symposium in 1962 (Heineman and Jensen, 2006). The first commercially produced biosensor Springs Instruments (Yellow Springs, OH, USA) placed on the market in 1975 (Pohanka and Skladal, 2008). Aflatoxin has inhibitory effect on acetylcholinesterase (AchE) and its detection is coupled with the decrease in the activity of AchE which is measured using a choline oxidase amperometric biosensor. (Ben Rejeb et al., 2009). Early studies found that Aflatoxin production by Aspergillus flavus is reduced when it is cultivated with certain other fungi and bacteria (Ashworth et al., 1965; Weckbach and Marth, 1977; Wicklow et al., 1980, 1988; Horn and Wicklow, 1983; Ehrlich et al., 1985). These organisms produce certain myco-toxin degrading enzymes. Flavobacterium auranticum first micro- organism with myco-toxin degradation activity was isolated in 1966 (Ciegler et al., 1966). Detoxifying agents (adsorbing agents and bio-transforming agents) is one of the well-known strategies for the management of aflatoxins in foods and feeds in Table 4.

\section{Biosensor}

A biosensor is an analytical device, used for the detection of an analyte, that combines a biological component with a physicochemical detector (Turner et al., 1987, Bănică, 2012).

\section{Components of biosensor}

Sensitive biological element: A biologically derived material or biomimetic component that interacts the analyte under study e.g. tissue, microorganisms, organelles, cell receptors, artificial receptors, enzymes, antibodies, nucleic acids, etc. (URL1).

Transducer/ detector element: is transform the signal resulting from the interaction of the analyte with the biological element into another signal that can be more easily measured and quantified. Transducer works in a physicochemical way; Electro-chemical, optical, electronic, piezoelectric, gravimetric and pyro-electric (Li et al., 2011).

Biosensor reader device: is associated electronics or signal processors that are primarily responsible for the display of the results in a user friendly way. Known manufacturers of biosensor electronic readers include Palm Sens, Gwent Biotechnology Systems and Rapid Labs (Cavalcanti et al., 2008).

\section{Classification of biosensors based on type of bio-transducer}

Biosensors can be classified by their biotransducer type (URL1) in Table 2. The most common types of bio-transducers used in 
biosensors are1) Electrochemical biosensors, 2) Optical biosensors, 3) Electronic biosensors, 4) Piezoelectric biosensors, 5) Gravimetric biosensors, 6) Pyro-electric biosensors

Electrochemical biosensors: are normally based on enzymatic catalysis of a reaction that produces or consumes electrons. The sensor substrate usually contains three electrodes; a reference electrode, a working electrode and a counter electrode. Measures rate of flow of electrons that is proportional to the analyte concentration at a fixed potential. Potential of the working electrode is space charge sensitive and this is often used. Further, the label free and direct electrical detection of small peptides and proteins is possible by their intrinsic charges using bio-functionalized ion sensitive field effect transistors (Lud et al., 2006).

Principle: Based on their operating principle, the electrochemical biosensors can employ potentiometric, amperometric and impedimetric transducers converting the chemical information into a measurable amperometric signal. In electrochemistry, the reaction under investigation generate a measurable current (amperometric), a measurable potential or charge accumulation (potentiometric) or measurably alter the conductive properties of a medium (conductimetric) between electrodes (Tothill and Turner, 2003). Cottrell equation can indicate this situation better here-

$I=n F A c_{o} \sqrt{ } \frac{D}{\pi t}$

If, $I$ : current is dependent on, $F$ : Faraday's constant, $n$ : The number of transferred electrons for each molecule, $A$ : The electrode area, $c_{0}$ : The analyte concentration, $D$ : The diffusion coefficient and $t$ : Time (Koyun et al., 2012).

Cryptogram: Measured Parameter> Applied

\section{Voltage> Sensitivity> Governing Equation> Fabrication}

Amperometric: Current> Constant potential (DC)> High> Cottrell Equation> FET+ Enzyme 2 electrodes

Conductimetric: Conductance/ Resistance> Sinusoidal (AC)> Low> Incremental resistance> FET+ Enzyme

Potentiometric: Potential/ Voltage> Ramp Voltage> High> Nernst Equation> FET+ Enzyme Oxide electrode

Optical biosensors: An optical biosensor, the transduction process induces a change in the phase, amplitude, polarization and frequency of input light in response to the physical or chemical change produced by the biorecognition process (Lading et. al., 2002; Bosch et. al., 2007). The sensor works by sending light through an optical fiber to the bio sample. The amount of light absorbed by the analyte is determined by measuring the light coupled out via second optical fiber. Catalytic and affinity reactions can be measured (Biran and Walt, 2004). The main components of an optical biosensor are light source, optical transmission medium (fiber, waveguide etc.), immobilized biological recognition elements (enzymes, antibodies, or microbes) and optical detection system (Ligler and Taitt, 2008; Borisov and Wolfbeis (2008). Pharmacia Biosensors AB (Sweden) marketed a device called BIAcore, which measures antibody-antigen interactions for the study of drug-receptor interactions (Tothill, 2011).

Principle: The reaction causes a change in fluorescence or absorbance resulting due to change in the refractive index of the surface between two media which differ in density. Based on the Lambert-Beer's law stated that "absorbance is proportional to the concentrations of the attenuating species in the material sample" in 1852 (Valeur, 2001). 
Lambert-Beer's law is used for the absorption here-

$\mathrm{A}=\log (\mathrm{Io} / \mathrm{I})=\varepsilon .[\mathrm{C}] .1$

If, $\mathrm{A}=$ Optical absorbance, $\mathrm{I} 0=$ incident light intensity, I= transmitted light intensity, l=effective path length, $\varepsilon=$ Molar absorption coefficient (Koyun et al., 2012).

There are two type detection protocols-

\section{Fluorescence-based detection:}

Fluorescence protein (FP)-based biosensors: The sensing element consists of one or more polypeptide chains. The polypeptide chain acts as the molecular recognition element (MRE) that under goes conformational changes upon binding with analyte, producing a change in fluorescence properties (Nasir and Jolley, 2002). There are three type of FP- based biosensor based on their structure-

Fluorescence resonance energy transfer (FRET)- based biosensor: FRET describe the energy transfer between two chromophores. A doner chromophore, in a higher energy state, may transfer energy to an acceptor chromophore through nonradiative dipoledipole coupling. In FRET- based biosensors, two fluorescent proteins are genetically linked either to each end of a polypeptide chain i.e molecular recognition element (MRE) or two seprate polypeptides, the MRE and analyte protein. Change in the fluorescence intensities of the donor and acceptor FPs, which is measured in FRET efficiency (Chandran et al., 2015; Piatkevich and Verkhusha, 2010; Jares-Erijman and Jovin, 2003).

Bio-molecular

fluorescence complementation (BiFC) - based biosensor: Used to visualize a variety of protein- protein interactions in live cells. FP is split up and MRE are linked to one portion and the analyte protein is linked to other portion. Two proteins fuse together, refolding properly into three dimensional structures and producing a fluorescence signal (Kerppola, 2008a, b, Kodama and Wada, 2009, Chandran et al., 2015).

Single FP-based biosensor: A single fluorescent protein coupled with an MRE causes conformational changes of the fluorescent protein consequently altering its fluorescent properties ( $\mathrm{Su}$ et al., 2012, Ibraheem and Campbell, 2010).

\section{Chemiluminescence-based biosensors}

Reaction between analyte and immobilized biomolecule that has been marked with Chemiluminescence species will end in generating light as result of biochemical reaction. Emitted light can be detected using a photo multiplier tube (PMT) (Aboul-Enein et al., 2000; Gübitz et al., 2001).

\section{Label- free detection}

SPR-based biosensor: Use surface plasmon wave (electromagnetic wave) to detect changes when the target analyte interacts with the bio-recognition element on the sensor. SPR first observed by Wood (Wood, 1902, 1912) but SPR-based sensors first demonstrated by Liedberg et al., (1983).

Electronic biosensors: Ions or electrons are produced during the course of biochemical reaction, the overall conductivity or resistivity of the solution is changing. Transducer measured the electrical conductance/resistance of the solution. Conductance measurements have relatively low sensitivity. Using a sinusoidal voltage (AC) the electric field is generated which finally minimize undesirable effects such as Faradaic processes, double layer charging and concentration polarization (Mohanty and Kougianos, 2006). The inverse value of resistance is called conductance and thus the name conductometric has been used. 
Novel trends in case of impedimetric biosensors were reviewed by Guan et al., (2004) and the use of conductometric biosensors for biosecurity by MuhammadTahir and Alocilja (2003).

Principle: The Faradaic current is the current generated by the reduction or oxidation of some chemical substance at an electrode. The net faradaic current is the algebraic sum of all the faradaic currents flowing through an indicator electrode or working electrode. These biosensors are operated by field-effect modulation of carriers in a semiconductor due to nearby charged (Dzyadevych. et al., 2006, Rastislav et al., 2012).

Piezoelectric/ Acoustic biosensors: The first scientific experiment of piezoelectricity was conducted in 1880 by Pierre and Paul Jacques curie, who found that compression of various crystals leads to voltage production on their surface (Manbachi and Cobbold, 2011). These type of biosensors have two components; a receptor and a detector (transducers). The receptor is responsible for the selectivity of the sensor. Examples include enzymes, antibodies, and lipid layers. The detector, which plays the role of the transducer, translates the physical or chemical change by recognizing the analyte and relaying it through an electrical signal (Milstein and Das, 1977, $\mathrm{Li}$, 2008.). There are two types of piezoelectric sensors-

Bulk acoustic wave piezoelectric sensors: Transmit an acoustic wave from one crystal face to another (Kim et al., 2007; Fohlerová et al., 2007).

Surface acoustic wave piezoelectric sensors: Transmit an acoustic wave along a single crystal face from one location to another (Bowers et al., 1991, Guntupalli et al., 2007). The resonance frequency of these acoustic waves depends on physical properties of piezoelectric materials and these physical properties changes i e. mass, elasticity, conductivity and dielectric properties from mechanical or electrical variations. When antibodies are coated or when antigens bind to these antibodies. Antibodies lead to increase in mass (Liliana et al., 2011).

Principle: The basic equations describing the relationship between the resonance frequency of an oscillating piezoelectric crystal and the mass deposited on the crystal surface have been derived by Sauerbrey (Lostis, 1958; Sauerbrey, 1959, Stockbridge, 1996). In 1959, Sauerbrey developed an empirical equation for AT-cut quartz crystals vibrating in the thickness shear mode that describes the relationship between the mass of thin metal films deposited on quartz crystals and the corresponding change in resonant frequency of the crystal:

$\Delta \mathrm{F}=-2.3 \times 10^{6} \mathrm{~F}^{2}=\frac{\Delta \mathrm{M}}{\mathrm{A}}$

Where, $\Delta \mathrm{F}=$ frequency change in oscillating crystal in $\mathrm{Hz}, \mathrm{F}=$ frequency of piezoelectric quartz crystal in $\mathrm{MHz}, \Delta \mathrm{M}=$ mass of deposited film in $\mathrm{g}$, and $\mathrm{A}=$ area of electrode surface in $\mathrm{cm}^{2}$.

Gravimetric biosensors: are based on mass variation induced by an enzymatic reaction. Enzyme are proteic nature often fragile and instable results in a decrease in the enzyme activity and consequently in a decrease in the biosensor performances (Patolsky et al., 1999) in Table 1.

Principle: Gravimetric sensing is based on the fact that when the device mass increases, the resonant frequency of the device decreases. When mass attaches to the sensing surface, the frequency change of the resonant peak is detected. A higher quality factor allows for better resolution of detection (Shuchen et al., 2013; Walton et al., 1993).

Pyro-electric biosensors/ calorimetric 
biosensors: Calorimetric biosensors measure the change in temperature of the solution containing the analyte, following enzyme action and interpret it in terms of the analyte concentration in the solution (Maskow et al., 2012; Kirchner et al., 2012). The sensitivity $\left(10^{-4} \mathrm{M}\right)$ and the range $\left(10^{-4}-10^{-2} \mathrm{M}\right)$ of such biosensors are quite low for most applications. Calorimetric technique is capable of rapidly detecting the DNA hybridization.

Principle: Calorimetrics measured the changes in temperature in the reaction between bio-recognition element and a suitable analyte. Change in temperature can be correlated to amount of reactants consumed or products formed (Xie et al., 1999).

\section{Current biosensor research}

1. Identification of volatile marker compounds for biosensors.

3. Microsensor array for mycotoxin analysis.

4. Food pathogen biosensor scientists are inventing a new ways to protect the food supply from potentially deadly food pathogens.

5. Phosphate ion biosensor.

6. Biosensor has been developed for detection of insecticide, herbicide and their influence on the environment.

7. Wheat flour quality sensor to determine the starch damage and diacritic activity of wheat flour by the use of FIA biosensor (Hideaki et al., 2003).

\section{Application of bio-sensors}

- Measurement of product quality such as, aroma, taste, nutritional value, functional properties, compliance with specifications (Turner, 1989).

- Screening for product safety include, chemical contaminations, residues, toxins taints, microbial contamination, total load, pathogens, indicators of activity (Rogers, 1995).

- Assessing product stability towards, water activity, solute concentration, $\mathrm{pH}$ value, preservative concentration, protective atmosphere composition, chemical reaction and microbial growth (Lowe, 1985).

- Biosensors that use whole cells or enzymes have been used for the detection of alcohol (Valach at al., 2009).

Table.1 Surface area to mass ratio comparison for various gravimetric sensors

\begin{tabular}{|l|c|c|c|c|c|}
\hline Device & $\begin{array}{c}\text { Mass } \\
(\mathbf{g})\end{array}$ & $\begin{array}{c}\text { S.A. } \\
\left(\mathbf{m m}^{\mathbf{2}}\right)\end{array}$ & $\begin{array}{c}\text { Thickness } \\
(\boldsymbol{\mu m})\end{array}$ & $\begin{array}{c}\text { Operating } \\
\text { Frequency(Hz) }\end{array}$ & $\begin{array}{c}\text { S.A. to mass } \\
\text { ratio }\left(\mathbf{c m}^{2} / \mathbf{g}\right)\end{array}$ \\
\hline $\begin{array}{l}\text { SAW } \\
\text { (Bowers, et al. 1991) }\end{array}$ & - & 10 & - & $200 \times 10^{6}$ & 452 \\
\hline $\begin{array}{l}\text { QCM (Heineman and } \\
\text { Jensen, 2006) }\end{array}$ & $0.14 \mathrm{~g}$ & 154 & 300 & $5 \times 10^{6}$ & 11 \\
\hline $\begin{array}{l}\text { FPW(Clark et al. } \\
\text { 2002) }\end{array}$ & $15 \times 10^{-3}$ & 600 & 25 & $2-30$ & 400 \\
\hline $\begin{array}{l}\text { MEMS membrane } \\
\text { (Bartkovsky et al, } \\
\text { 2004) }\end{array}$ & $\begin{array}{c}94.4 \mathrm{x} \\
10^{-9}\end{array}$ & 0.02 & 1.65 & $200 \times 10^{3}$ & 2120 \\
\hline
\end{tabular}


Table.2 Classification of biosensors based on type of bio-transducer

\begin{tabular}{|c|c|c|c|c|}
\hline Mycotoxin & Matrix & Biosensor principle & $\begin{array}{l}\text { Limit of } \\
\text { Detection }\end{array}$ & Reference \\
\hline \multirow[t]{2}{*}{ Aflatoxin B1 } & $\begin{array}{l}\text { Maize, } \\
\text { sorghum, nuts }\end{array}$ & Optical & $5 \mu \mathrm{g} \mathrm{kg}^{-1}$ & Nasir \& Jolley (2002) \\
\hline & $\begin{array}{l}\text { Barley } \\
\text { Corn }\end{array}$ & $\begin{array}{l}\text { Electrochemical } \\
\text { QCM }\end{array}$ & $\begin{array}{l}0.03 \mu \mathrm{g} \mathrm{kg}^{-1} \\
0.03 \mu \mathrm{g} \mathrm{L}^{-1}\end{array}$ & $\begin{array}{l}\text { Ammida et al.,(2004) } \\
\text { Piermarini et } \\
\text { al.,(2007) }\end{array}$ \\
\hline Aflatoxin M1 & $\begin{array}{l}\text { Milk } \\
\text { Milk } \\
\text { Milk } \\
\text { Milk } \\
\text { Milk }\end{array}$ & Electrochemical & $\begin{array}{l}0.02 \mu \mathrm{g} \mathrm{L}^{-1} \\
0.05 \mu \mathrm{g} \mathrm{L}^{-1} \\
0.039 \mu \mathrm{g} \mathrm{L}^{-1} \\
0.008 \mu \mathrm{g} \mathrm{L}^{-1} \\
0.01 \mu \mathrm{g} \mathrm{L}^{-1}\end{array}$ & $\begin{array}{l}\text { Badea } \text { et al.,(2004) } \\
\text { Micheli at al., (2005) } \\
\text { Parker \& Tothill } \\
\text { (2009) } \\
\text { Parker } \text { et al.,(2009) } \\
\text { Paniel } \text { et al.,(2010) }\end{array}$ \\
\hline $\begin{array}{l}\text { Fumonisin } \\
\text { B1 }\end{array}$ & $\begin{array}{l}\text { Corn } \\
\text { Corn } \\
\text { Corn }\end{array}$ & $\begin{array}{l}\text { Optical } \\
\text { Electrochemical }\end{array}$ & $\begin{array}{l}50 \mu \mathrm{g} \mathrm{kg}^{-1} \\
10 \mu \mathrm{g} \mathrm{L}^{-1} \\
5 \mu \mathrm{g} \mathrm{L}^{-1}\end{array}$ & $\begin{array}{l}\text { Mullett et al.,(1998) } \\
\text { Thompson \& Maragos } \\
\text { (1996), Abdul Kadir \& } \\
\text { Tothill (2010) }\end{array}$ \\
\hline Ochratoxin A & $\begin{array}{l}\text { Cereals } \\
\text { Wheat } \\
\text { Wine } \\
\text { Wine }\end{array}$ & $\begin{array}{l}\text { Optical } \\
\text { Electrochemical } \\
\text { QCM }\end{array}$ & $\begin{array}{l}3.8 \text { to } 100 \mu \mathrm{g} \\
\mathrm{kg}^{-1} \\
0.06 \mu \mathrm{g} \mathrm{L}-1 \\
0.01 \mu \mathrm{g} \mathrm{L}^{-1} \\
16.1 \mu \mathrm{g} \mathrm{L}^{-1}\end{array}$ & $\begin{array}{l}\text { Ngundi } \text { et al.,(2005) } \\
\text { Alarcon } \text { et al.,(2006) } \\
\text { Heurich } \text { et al.,(2011) } \\
\text { Tsai and Hsieh (2007) }\end{array}$ \\
\hline Deoxynivalenol & $\begin{array}{l}\text { Wheat } \\
\text { Wheat } \\
\text { Oats }\end{array}$ & Optical & $\begin{array}{l}2.5 \mu \mathrm{g} \mathrm{L}-1 \\
2.5 \mu \mathrm{g} \mathrm{L}-1 \\
50 \mu \mathrm{g} \mathrm{kg}-1\end{array}$ & $\begin{array}{l}\text { Schnerr } \text { et al.,(2002) } \\
\text { Tudos } \text { et al.,(2003) } \\
\text { Ngundi et al.,(2006) }\end{array}$ \\
\hline $\begin{array}{l}\alpha \text {-zearalenol } \\
\alpha \text {-zearalanol } \\
\text { Zearalanone } \\
\text { Zearalenone } \\
\beta \text {-zearalanol } \\
\beta \text {-zearalenol } \\
\end{array}$ & $\begin{array}{l}\text { Milk } \\
\text { Milk } \\
\text { Milk } \\
\text { Milk } \\
\text { Milk } \\
\text { Milk }\end{array}$ & Electrochemical & $\begin{array}{l}0.001 \mu \mathrm{g} \mathrm{L}^{-1} \\
0.001 \mu \mathrm{g} \mathrm{L}^{-1} \\
0.002 \mu \mathrm{g} \mathrm{L}^{-1} \\
0.009 \mu \mathrm{g} \mathrm{L}^{-1} \\
0.015 \mu \mathrm{g} \mathrm{L}^{-1} \\
0.071 \mu \mathrm{g} \mathrm{L}^{-1}\end{array}$ & Välimaa et al.,(2010) \\
\hline
\end{tabular}


Table.3 Myco-toxins: Types, classification, food commodity, action level, mode of action and pathological effects

\begin{tabular}{|c|c|c|c|c|c|c|c|}
\hline $\begin{array}{l}\text { S } \\
\text { No. }\end{array}$ & Mycotoxins & $\begin{array}{l}\text { Type(IARC } \\
\text { Classification) }\end{array}$ & Fungal Species & Food commodity & Action level & Mode of action & Pathological Effects \\
\hline 1. & $\begin{array}{l}\text { Aflatoxins } \\
(6 \mathrm{~T}) \quad \text { (Post- } \\
\text { harvest) }\end{array}$ & $\begin{array}{l}\mathrm{AFB}_{1}(1) \mathrm{C}_{17} \mathrm{H}_{12} \mathrm{O}_{6}, \mathrm{MW}= \\
312, \quad \mathrm{AFB}_{2}(1) \quad \mathrm{C}_{17} \mathrm{H}_{14} \mathrm{O}_{6}, \\
\mathrm{MW}=314, \quad \mathrm{AFG}_{1}(1) \\
\mathrm{C}_{17} \mathrm{H}_{12} \mathrm{O}_{7}, \quad \mathrm{MW}=\quad 328, \\
\mathrm{AFG}_{2}(1) \mathrm{C}_{17} \mathrm{H}_{14} \mathrm{O}_{7}, \mathrm{MW}= \\
330, \\
\mathrm{AFM}_{1}(2 \mathrm{~B}) \& \mathrm{AFM}_{2}(2 \mathrm{~B})\end{array}$ & $\begin{array}{l}\text { Aspergillus flavus, A. nomius, A. parasiticus, } \\
\text { A. arachidicola, A. bombycis, A. } \\
\text { pseudotamarii, A. minisclerotigenes, A. } \\
\text { rambellii, A. ochraceoroseus, Emericell } \\
\text { astellata, E. venezuelensis, E. olivicola } \\
\text { (CAST, 2003). }\end{array}$ & $\begin{array}{l}\text { Maize, wheat, rice, spices, } \\
\text { sorghum, groundnut, } \\
\text { treenut, almonds, milk, } \\
\text { oilseeds, dried fruit, } \\
\text { cheese, eggs and meat } \\
\text { (D'Mello, 2003). }\end{array}$ & $\begin{array}{l}\mathrm{AFB}_{1} \\
\mathrm{AFB}_{2}=<20 \\
\mathrm{ppb} . \\
\mathrm{AFM}_{1} \quad \& \\
\mathrm{AFM}_{2-}<0.5 \\
\text { ppb } \\
\text { (Christopher } \\
\text { and Gong, } \\
\text { 2010). }\end{array}$ & $\begin{array}{l}\text { Cytochrome P450 enzymes convert } \\
\text { aflatoxins to the reactive } 8,9 \text {-epoxide form } \\
\text { which is capable of binding to both DNA } \\
\text { and proteins. Aflatoxin B1-DNA adducts } \\
\text { can result in GC to TA transversions (Raj } \\
\text { et al.,1986) }\end{array}$ & $\begin{array}{l}\text { Carcinogenic, mutagenic, } \\
\text { teratogenic, heptotoxic, } \\
\text { nephrotoxic, } \\
\text { immunosuppressive, } \\
\text { hemorrhage of intestinal tract } \\
\text { and kidney, liver disease } \\
\text { (Christopher and Gong, } \\
\text { 2010). }\end{array}$ \\
\hline 2 & $\begin{array}{l}\text { Fumonisins } \\
(6 \mathrm{~T})(\text { Pre- } \\
\text { harvest) }\end{array}$ & $\begin{array}{lr}\mathrm{FB}_{1}(2 \mathrm{~B}), & \mathrm{FB}_{2}(2 \mathrm{~B}), \\
\mathrm{FB}_{3}(2 \mathrm{~B}), & \mathrm{FB}_{4}(2 \mathrm{~B}) \\
\mathrm{FA}_{1}(2 \mathrm{~B}) \& \mathrm{FA}_{2}(2 \mathrm{~B})\end{array}$ & $\begin{array}{l}\text { Alternaria alternate, Fusarium anthophilum, } \\
F . \text { moniliforme, F.dlamini, } F \text {. napiforme, } F . \\
\text { proliferatum, F. nygamai, F. verticillioides } \\
\text { (Lukasz, 2014, Picot et al. 2010) }\end{array}$ & $\begin{array}{l}\text { Maize, maize based } \\
\text { products, corn based } \\
\text { products, sorghum, } \\
\text { asparagus, rice and milk } \\
\left(D^{\prime \prime} \text { Mello, 2003). }\right.\end{array}$ & $\begin{array}{l}<2000 \text { ppb } \\
\text { (Christopher } \\
\text { and Gong, } \\
\text { 2010). }\end{array}$ & $\begin{array}{l}\text { Interfering with sphingolipid metabolism } \\
\text { (Merrill et al., 2001) }\end{array}$ & $\begin{array}{l}\text { Hepatotoxic, cerebral edema, } \\
\text { necrosis, immunotoxic } \\
\text { (Christopher and Gong, } \\
\text { 2010). }\end{array}$ \\
\hline 3 & $\begin{array}{l}\text { Ochratoxin } \\
\text { (Post-harvest) }\end{array}$ & $\begin{array}{l}\text { OTA(2B) } \quad \mathrm{C}_{20} \mathrm{H}_{18} \mathrm{O}_{6} \mathrm{NC}_{\mathrm{l}}, \\
\text { OTB(2B), OTC(2B) }\end{array}$ & $\begin{array}{l}\text { A. alutaceus, A. alliaceus, A. auricomus, A. } \\
\text { glaucus, A. niger, A. carbonarius, A. melleus, } \\
\text { A. albertensis, A. citricus, A. flocculosus, A. } \\
\text { fonsecaeus, A. lanosus, A. ochraceous, A. } \\
\text { ostianus, A. petrakii, A. sulphureus, A. } \\
\text { pseudoelengans, A. roseoglobulosus, A. } \\
\text { sclerotiarum, A. steynii, A. westerdijkiae, } \\
\text { Neopetromyces muricatus, Penicillium } \\
\text { viridicatum, P. verrucosum, P.cyclopium and } \\
\text { P. carbonarius (D'Mello, 2003). }\end{array}$ & $\begin{array}{l}\text { Cereals, dried vine fruit, } \\
\text { wine coffee, oats, spices, } \\
\text { rye, raisins and grape } \\
\text { juice Cereals, dried vine } \\
\text { fruit, wine coffee, oats, } \\
\text { spices, rye, raisins and } \\
\text { grape juice (Halasz et } \\
\text { al.,2009) }\end{array}$ & $\begin{array}{l}\text { OTA }=<5 \\
\text { ppb } \\
\text { (Murphy, et } \\
\text { al. 2006) }\end{array}$ & $\begin{array}{l}\text { Inhibits the enzyme involved in the } \\
\text { synthesis of the phenylalanine tRNA } \\
\text { complex (Bunge et al.,1979) (Marquardt } \\
\text { and Frohlich, 1992). In addition, it inhibits } \\
\text { mitochondrial } \\
\text { ATP production (Meisner and Meisner, } \\
\text { 1981). }\end{array}$ & $\begin{array}{l}\text { Carcinogenic, mutagenic, } \\
\text { teratogenic, heptotoxic, } \\
\text { nephrotoxic, } \\
\text { immunodeppressants and } \\
\text { inhibition of protein synthesis } \\
\text { (Thrasher and Crawley, } \\
\text { 2009). }\end{array}$ \\
\hline 4 & $\begin{array}{l}\text { Trichothecenes } \\
\text { (Pre-harvest) }\end{array}$ & 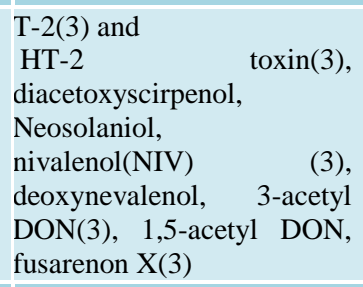 & $\begin{array}{l}\text { Fusarium sporotrichioides, F. poae, } F . \\
\text { acuminatum, F. culmorum, F. equiseti, } F . \\
\text { graminearum, F. cerealis, F.moniliforme, F. } \\
\text { myrothecium, } \quad F . \quad \text { lunulosporum, } \\
\text { Cephalosporium sp. (D'Mello, 2003). }\end{array}$ & $\begin{array}{l}\text { Cerial and cerial based } \\
\text { products } \\
\text { 2003). }\end{array}$ & $\begin{array}{l}\mathrm{T}-2 \quad=<100 \\
\text { ppb, } \\
\mathrm{DON}=<1000 \\
\text { ppb(Murphy } \\
\text { et al., 2006) } \\
\text { NIV }=<0.2 \\
\text { ppm }\end{array}$ & $\begin{array}{l}\text { Acting both on the cell immune system and } \\
\text { on the number of macrophages, } \\
\text { lymphocytes and erythrocytes. T-2 and } \\
\text { deoxynivalenol (DON) are known to } \\
\text { inhibit protein synthesis and cause cell } \\
\text { death in various } \\
\text { parts of the body. Upadhaya et al., (2010) }\end{array}$ & $\begin{array}{l}\text { Immuno-depressants, } \\
\text { mutagenic, gastrointestinal } \\
\text { haemorrhaging } r \text { and } \\
\text { neurotoxic (Thrasher and } \\
\text { Crawley, 2009). }\end{array}$ \\
\hline 5 & $\begin{array}{l}\text { Zearalenone } \\
\text { (Pre-harvest) }\end{array}$ & $\begin{array}{l}\alpha-\mathrm{ZON}(3) \& \\
\beta-\mathrm{ZON}(3)\end{array}$ & 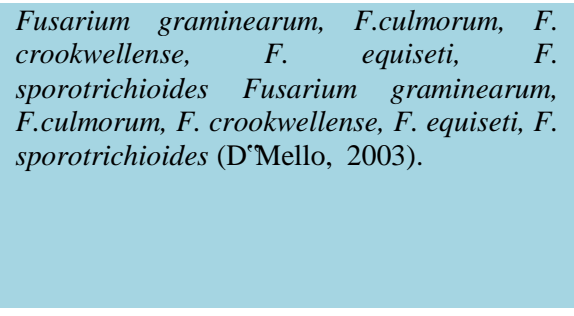 & $\begin{array}{l}\text { Barley, oats, wheat, rice } \\
\text { sorghum, sesame, } \\
\text { soybeans and cereal } \\
\text { based products } \\
\text { (D'Mello, 2003). }\end{array}$ & $\begin{array}{l}<1 \mathrm{ppm} \\
\text { (Abdelhamid, } \\
\text { 1990). }\end{array}$ & $\begin{array}{l}\text { Binds to oestrogen } \\
\text { receptors binds to oestrogen receptors } \\
\text { (Zinedine et al., 2007). Decrease in the } \\
\text { amounts of luteinizing hormone (LH) and } \\
\text { progesterone produced affecting the } \\
\text { morphology of uterine tissues, decrease in } \\
\text { milk production, feminization of young } \\
\text { males due to decreased testosterone } \\
\text { production(Guerre } \text { et al.,2000) }\end{array}$ & $\begin{array}{l}\text { Estrogenic activity (infertily, } \\
\text { vulval oedema, vaginal } \\
\text { prolapsed, mammary } \\
\text { hypertrophy in females, } \\
\text { feminisation of males } \\
\text { (Thrasher and Crawley, } \\
\text { 2009). }\end{array}$ \\
\hline
\end{tabular}

Note: $1 \mu \mathrm{g} / \mathrm{kg}=1 \mathrm{ppb}, 1 \mathrm{ppm}=1000 \mathrm{ppb}, 1 \mathrm{mg} / \mathrm{kg}=1 \mathrm{ppm}$ 
Table.4 Detoxifying agents: absorbing and Bio-transforming agents and their product, dose, target myco-toxin and company based on available literature

\begin{tabular}{|c|c|c|c|c|}
\hline $\begin{array}{l}\text { S. } \\
\text { No. }\end{array}$ & Product & Dose & $\begin{array}{l}\text { Targeted } \\
\text { myco-toxin }\end{array}$ & Company \\
\hline A. & \multicolumn{4}{|l|}{ Mycotoxin-adsorbing agents } \\
\hline 1. & $\begin{array}{l}\text { Astra Ben } 20 \AA \text { (sodium } \\
\text { bentonite), Red Crown } \AA \\
\text { (calcium bentonite) }\end{array}$ & $227 \mathrm{~g} / \mathrm{cow}$ daily & AFB1, AFM1 & $\begin{array}{l}\text { Prince Agri-products } \\
\text { (Diaz et al.,2004) (Kissell et al. } \\
\text { 2013) }\end{array}$ \\
\hline 2. & $\begin{array}{l}\text { Flow Guard® (sodium } \\
\text { bentonite) }\end{array}$ & $1.1 \%$ of $\mathrm{DM}$ & AFB1, AFM1 & $\begin{array}{l}\text { Laporte Biochem, Inc. } \\
\text { (Diaz et al.,2004) }\end{array}$ \\
\hline 4. & $\begin{array}{l}\text { Milbond-TX®: inert } \\
\text { montmorillonite clay-based } \\
\text { adsorbing agent }\end{array}$ & $2.0 \%$ of $\mathrm{DM}$ & AFB1 & $\begin{array}{l}\text { Milwhite Inc. (Marroquin-Cardona } \\
\text { et al.,2009) }\end{array}$ \\
\hline 5. & NovaSilTM $^{\text {TM }}$ & 1 to $5 \mathrm{~kg} / \mathrm{t}$ of feed & AFB1, AFM1 & $\begin{array}{l}\text { Engelhard Chemical corporation } \\
\text { (Galvano et al.,1996), (Jaynes et } \\
\text { al.,2007), (Moschini et al.,2008), } \\
\text { (Wang et al.,2008) }\end{array}$ \\
\hline 8. & Filtrasorb 400 & $2 \%$ & Aflatoxin & $\begin{array}{l}\text { Calgon carbon corporation (Di } \\
\text { Natale et al.,2009) }\end{array}$ \\
\hline 9. & Nuchar® SA-20 & $2 \%$ & AFB1, AFM1 & Westvaco (Diaz et al.,2004) \\
\hline 10 & $\begin{array}{l}\text { Mycosorb }{ }^{\mathrm{TM}}, \mathrm{MTB}-100 \AA \\
\text { (polymeric glucomannan } \\
\text { adsorbing agent extracted } \\
\text { from the cell wall of yeast) }\end{array}$ & $0.05-0.2 \%$ & $\begin{array}{l}\text { OTA, FB1, } \\
\text { Moniliformin, } \\
\text { ZEA, AFB1, } \\
\text { AFM1, T-2 toxin, } \\
\text { DAS, DON, NIV, }\end{array}$ & $\begin{array}{l}\text { Alltech (Bursian, 2004), (Diaz et } \\
\text { al.,2004), (Chowdhury and Smith, } \\
\text { 2005), (Kogan and Kocher, 2007), } \\
\text { (Dvorska, 2007), (Smith et } \\
\text { al.,2008), (Moschini et al.,2008), }\end{array}$ \\
\hline
\end{tabular}




\begin{tabular}{|c|c|c|c|c|}
\hline & & & fusaric acid & (Meissonnier et al.,2009) \\
\hline 11 & \begin{tabular}{lrrr} 
Lactobacillus & \multicolumn{2}{r}{ rhamnosus } \\
strain GG & \multicolumn{2}{r}{ Lactobacillus } \\
helveticus & 46 & and & 72 \\
Lactobacillus & jugurti & 63 \\
Lactobacillus & lactis & 170 \\
Lactobacillus & casei & spp. \\
Casei C3 & Streptococcus \\
thermophilus & NG40Z and C5 \\
\multicolumn{2}{l}{ Lactobacillus paraplantarum }
\end{tabular} & $\begin{array}{l}1.7 \times 10^{8} \text { to } 2.2 \times 10^{9} \mathrm{cfu} \mathrm{kg-}^{1} \text { of } \\
\text { complete feedstuff }\end{array}$ & $\begin{array}{l}\text { DON, FB1, FB2, } \\
\text { ZEA }\end{array}$ & $\begin{array}{l}\text { Promochem INRA Thivernal- } \\
\text { Grignon ( Niderkorn } \text { et al.,2006), } \\
\text { (Niderkorn } \text { et al.,2007), (Niderkorn } \\
\text { et al.,2008), (Niderkorn } \text { et al.,2009) }\end{array}$ \\
\hline 12 & $\begin{array}{l}\text { Adfimax }{ }^{\circledR} 82 \text { (oat fibers) } \\
\text { Adfimax }{ }^{\circledR} 75 \text { (apple fibers) } \\
\text { Adfimax }{ }^{\circledR} 59 \text { (grape pulp } \\
\text { fibers) } \\
\text { Adfimax }{ }^{\circledR} 90 \text { (pea seed } \\
\text { fibers) } \\
\text { Adfimax }{ }^{\circledR} 84 \text { (lupine seed } \\
\text { fibers) } \\
\text { Adfimax }{ }^{\circledR} 80 \text { (lupine seed } \\
\text { fibers) } \\
\text { ( Micronized fibers) }\end{array}$ & $20 \mathrm{~g} / 1$ & OTA & $\begin{array}{l}\text { REALDYME (Tangni 2003, } \\
\text { (Tangni et al.,2005), (Aoudia et } \\
\text { al.,2008), (Aoudia et al.,2009) }\end{array}$ \\
\hline 13 & $\begin{array}{l}\text { Antitox Vana } \\
\text { (Polyvinylpolypyrrodilone, } \\
\mathrm{C}_{6} \mathrm{H}_{9} \mathrm{NO} \text { ) }\end{array}$ & $20 \mathrm{~g} / 1$ & DON & $\begin{array}{l}\text { Qualitech Products Inc. (Friend, } \\
\text { 1984) }\end{array}$ \\
\hline \multicolumn{5}{|c|}{ 2. Bio-transforming agents } \\
\hline 1. & $\begin{array}{l}\text { Biomin BBSH } 797 \\
\text { (Eubacterium s.p. BBSH } \\
\text { 797) }\end{array}$ & $\begin{array}{l}1.7 \times 10^{8} \text { to } 2.2 \times 10^{9} \mathrm{cfu} \mathrm{kg-}^{1} \text { of } \\
\text { complete feedstuff }\end{array}$ & $\begin{array}{l}\text { T-2 toxin, HT-2 } \\
\text { toxin, T-2 tetraol, } \\
\text { T-2 triol, } \\
\text { scirpentriol }\end{array}$ & Biomin (Fuchs et al.,2002) \\
\hline
\end{tabular}




\begin{tabular}{|c|c|c|c|c|}
\hline 2. & $\begin{array}{l}\text { Aspergillus niger, Eurotium } \\
\text { herbariorum, Rhizopus sp., } \\
\text { and non-aflatoxin (AF)- } \\
\text { producing A. flavus }\end{array}$ & $2 \mathrm{~g} / \mathrm{kg}$ of feed & AFB1, Aflatoxiol & (Nakazato et al., 1990) \\
\hline 4. & $\begin{array}{l}\text { Combination of Eubacterium } \\
\text { BBSH } 797 \text { and Trichosporon } \\
\text { mycotoxinivorans }\end{array}$ & $\begin{array}{l}2 \mathrm{~g}+1 \mathrm{~g} / \mathrm{kg} \text { of feed } \\
(\text { Karaman et al., 2005) }\end{array}$ & OTA, ZEA & Biomin (Hofstetter et al.,2006) \\
\hline 5. & $\begin{array}{l}\text { Amano" } 2 \text { SD (Protease A - } \\
\text { Aspergillus Niger) }\end{array}$ & $\begin{array}{l}2000 \text { units per } \mathrm{kg} \\
\text { (Xu et al., 2014) }\end{array}$ & OTA & Amano Inc. (Abrunhosa et al.,2006) \\
\hline
\end{tabular}




\section{Management of myco-toxin through detoxifying agents in feed}

Mycotoxin-detoxifying agents for reduction of the contamination: substances that can suppress or reduce the absorption, promote the excretion of mycotoxins or modify their mode of action". Depending on their mode of action, these feed additives may act by reducing the bioavailability of the mycotoxins or by degrading them or transforming them into less toxic metabolites Table 4. Therefore, we can define at least two main categories; including first various mycotoxin adsorbing agents leads to a reduction of mycotoxin uptake as well as distribution to the blood and target organs. Adsorbing agents are also called binding agents, adsorbents and second bio-transforming agents leads to degradation of mycotoxins into non-toxic metabolites; such as bacteria/fungi or enzymes (EFSA, 2002). Absorbing agents (AAs), on the basis of the toxicokinetic principle, since they can reduce mycotoxin absorption by the gastrointestinal tract, they can reduce the further steps such as toxin distribution and metabolism in organs and tissues: for example, the more mycotoxins such as AFB1 and OTA are sequestered, the less residues could be found in liver and kidneys. The consequence is also the decrease of residue and/or metabolite excretion in eggs for poultry and in milk for mammals and in particular mycotoxins: for example the more AFB1 is sequestered, the less AFM1 could be found in milk. Some AAs, have shown also other health benefits than those of counteracting the direct and indirect effects due to mycotoxins. The cell wall of Saccharomyces cerevisiae is composed of beta-D-glucans which have, besides their high binding properties towards ZEA and AFB1 and to a lesser extent to FB1 and DON, a well-known ability to stimulate the immune system (Yiannikouris et al., 2004). Regarding the ability of kaolin when given to animals in their diet, to bind, in addition to aflatoxins, heavy metals, plant metabolites, poisons, diarrhea-causing enterotoxins, and pathogenic microorganisms. Bentonites have been also found to be excellent adsorptive materials of heavy metals and bacteria. Bentonites including in particular montmorillonites, when used in animal diets, act as gut protectants (Trckova et al., 2004; Phillips et al., 2008). Since biotransforming agents are capable of modifying the molecular structure of some mycotoxins, the new conpound is supposed to be less toxic than the parent mycotoxin. If molecular structure(s) of new compound(s) is not modified during the toxicokinetic steps, the minimized toxicity is respected. Consequently, the action of biotransforming agents can improve the sanitary safety of some animal products.

In conclusions, there is a worldwide interest in innovation of the sensing receptor, the transducer and the accompanying electronics and analysis software and microfluidics. A single analyte testing to multianalyte analysis, miniaturization and novel materials such as nanoparticles and micro/nano based transducers are playing a major part in producing highly sensitive and cost effective devices. Current trends to produce chip-based micro/ nano-arrays for multi myco-toxins analysis are challenging. Nanoparticles of such as gold, silver, metal oxides and quantum dots assay developments will enhance the capability of the biosensor technology for myco-toxins analysis. In early, sensitive detection will aid in eliminating these toxins from entering the food chain and preventing ill health and protecting life. The chemical complexity of mycotoxins means that the effectiveness of an absorbing compound does not equalize ability to sequester one mycotoxin to other. Mycotoxinadsorbing agents can interact with vitamin or minerals other nutrients of feed. In this case, health benefits of these nutriments could be 
reduced. Biotransforming agents to be used in practice as animal feed additives must rapidly degrade mycotoxins into non-toxic metabolites, under different oxygen conditions and in a complex environment. They must be safe for animals and stable in the gastrointestinal tract. Therefore these innovative technologies need to be appropriate funding to move the technology from research to commercial products.

\section{References}

Abdelhamid, A.M., 1990. Arch. Anim. Nutr., 40, 647-664.

Abdul Kadir, M.K., Tothill, I.E., 2010. Toxins. 2, 382-398.

Aboul-Enein, H.Y., Stefan, R.I., Van Staden, J.F., Zhang, X.R., Garcia- Campana, A.M., Baeyens, W.R.G., 2000. Critical Rev. Anal. Chem. 30(4), 271-289.

Abrunhosa, L., Santos, L., Venancio, A., 2006. Food Biotechnology. 20, 231-242.

Alarcon, S.H., Palleschi, G., Campahnone, D., Pascale, M., Visconti, A., Barna-Vetro, I., 2006. Talanta 69: 1031-1037.

Ammida, N.H.S., Micheli, L., Palleschi, G., 2004. Analytica Chimica Acta. 520, 159-164.

Aoudia, N., Callu, P., Grosjean, F., Larondelle, Y., 2009. Food and Chemical Toxicology. 47, 1485-9.

Aoudia, N., Tangni, E.K., Larondelle, Y., 2008. Food and Chemical Toxicology, 46, 871-8.

Ashworth, L. J., Schroeder, H.W., Langley, B.C., 1965. Science. 148, 1228-1229.

Avantaggiato, G., Havenaar, R., Visconti, A., 2007. Journal of Agricultural and Food Chemistry. 55, 4810-9.

Avantaggiato, G., Solfrizzo, M., Visconti, A., 2005. Food Additives and Contaminants. 22(4), 379-88.

Badea, M., Micheli, L., Messia, M.C., Candigliota, T., Marconi, E., Mottram, T., Velasco-Garcia, M., Moscone, D. and Palleschi. G., 2004. Analytica Chimica Acta. 520, 141-8.

Banica F.G., 2012. Chemical Sensors and Biosensors: Fundamentals and Applications, Wiley publication. pp. 576

Bănică, F.G., 2012. Chemical Sensors and Biosensors: Fundamentals and Applications. Chichester, UK: John Wiley \& Sons. pp. 576.
Bartkovsky, M.J., Przybycien, T.M. and Hauan, S., 2004. Photochemical modification of a MEMS membrane device for use as a novel gravimetric based biosensor. AIChE annual meeting, Austin, TX, Advances in Biosensors I, 36.

Ben-Rejeb, I., Arduini,F., Arvinte, A., Amine, A., Gargouri, M., Micheli, L., Bala, C., Moscone, D., Palleschi, G., 2009. Biosensors and Bioelectronics, 24, 1962-8.

Biran, I., Walt, D.R., 2004. Optrode- Based Fiber Optic Biosensors (Bio-Optrode). In: Ligler F.S, Taitt C.A.R, editors. Optical Biosensors Present and Future. Elsevier, Amsterdam. pp 5-16.

Borisov, S.M., Wolfbeis, O.S., 2008. Chemical Reviews. 108, 423-461.

Bosch, M.E. et. al., 2007. Sensors. 7, 797-859.

Bove, F.J., 1970. The story of ergot. Basle: Karger Verlag.

Bowers, W., Chuan, R., Duong T., 1991. Review of Scientific Instruments. 62, 1624-9.

Bunge, I., Heller, K., Roschenthaler, R., 1979. Z. Lebensm. Unters. Forsch. 168, 457-8.

Bursian, S.J., Mitchell, R. R., Yamini, B., Fitzgerald, S. D., Murphy, P. A., Fernadez, G., Rottinghaus, G. E., Moran, L., Leefers, K., Choi, I., 2004. Veterinary and Human Toxicology. 46, 122-129.

Campbell, R.E., 2009. Anal. Chem, 81, 5972-9.

CAST, 2003. Mycotoxins: Risks in Plant, Animal, and Human Systems. Ames Council for Agricultural Science and Technology. Iowa, USA. January, 2003 Task Force Report No. 139.

Cavalcanti, A., Shirinzadeh, B., Zhang, M., Kretly, L.C. 2008. Sensors. 8 (5), 2932-58.

Chandran, Karunakaran; Bhargava Kalpana; Robson, Benjamin., 2015. Biosensors and Bioelectronics. Elsevier publisher, Amsterdam, Nitherland, pp. 344.

Chowdhury, S.R., Smith, T.K., 2005. Poultry Science. 84, 1671-4.

Christopher, P., Wild, Gong, Y.Y., 2010. Carcinogenesis. 31(1), 71-82.

Ciegler, A., Lillehoj, E. B., Peterson, R. E., Hall, H. H., 1966. Applied Microhiology. 14, 934-8.

Clark A., Whitehead L., Haynes C., Kotlicki, A., 2002. Novel resonant-frequency sensor to detect the kinetics of protein adsorption. Review of Scientific Instruments. 73, 433946.

D Mello, J.P.F., 2003. Mycotoxins in cereal grains, 
nuts and other plant products. In: Food safety contaminants and toxins. D'Mello JPF (Ed). Cromwell Press, Trowbridge, UK. pp. 65-90.

Dänicke, S., 2002. Journal of animal and feed sciences. 11, 437-51.

Dänicke, S., Gädeken, D., Ueberschär, K.H., Meyer, U., Scholz, H., 2002. Archives of Animal Nutrition/Archiv fur Tierernahrung. 56, 24561.

Dänicke, S., Matthes, S., Halle, I., Ueberschar, K.H., Döll, S., Valenta, H., 2003. British Poultry Science. 44, 113-26.

Di Natale, F., Gallo, M., Nigro, R., 2009. Adsorbents selection for aflatoxins removal in bovine milks. Journal of Food Engineering, -, - .

Diaz, D.E., Hagler, W.M., Blackwelder, J.T., Eve, J.A., Hopkins, B.A., Anderson, K.L., Jones, F.T., Whitlow, L.W., 2004. Aflatoxin binders II: Reduction of aflatoxin M1 in milk by sequestering agents of cows consuming aflatoxin in feed. Mycopathologia, 00, 1-8.

Diaz, D.E., Hagler, W.M., Hopkins, B.A., Whitlow, L.W., 2002. Aflatoxin binders I: In vitro binding assay for aflatoxin B1 by several potential sequestering agents. Mycopathologia, 156,223-226.

Diaz, G.J., Cortes, A., Roldan, L., 2005. Evaluation of the efficacy of four feed additives against the adverse effects of T-2 toxin in growing broiler chickens. Journal of Applied Poultry Research, 14, 226-231.

Dvorska, J.E., Pappas, A.C., Karadas, F., Speake, B.K., Surai, P.F., 2007. Comparative biochemistry and physiology. 145, 582-587.

Dzyadevych SV, Soldatkin AP, El'skaya AV, Martelet C, Jaffrezic-Renault N., 2006. Anal. Chim. Acta. 568, 248-258.

EFSA, 2002. The European Food Standard Agency Journal. 36, 1-179.

Ehrlich, K., Ciegler, A., Klich, M.A., Lee, L., 1985. Experientia. 41, 691-693.

Fohlerová, Z., Skládal, P., Turánek, J., 2007. Biosensors and Bioelectronics. 22(9-10), 1896-1901.

Friend, D.W., Trenholm, H.L., Young, J.C., Thompson, B.K., Martin, K.E., 1984. Canadian Journal of Animal Science. 64, 733741.

Fuchs, E., Binder, E.M., Heidler, D. and Krska, R., 2002. Structural characterization of metabolites after the microbial degradation of type A trichothecenes by the bacterial strain
BBSH 797. Food Additives and Contaminants 19: 379-386.

Galvano, F., Pietri, A., Bertuzzi, T., Fusconi, G., Galvano, M., Piva, A., Piva, G., 1996. Reduction of carryover of aflatoxin from cow feed to milk by addition of activated carbons. Journal of Food Protection, 59, 551-554.

Galvano, F., Pietri, A., Bertuzzi, T., Piva, A., Galvano, M., Chies, L., Galvano, M., 1998. Activated carbons: in vitro affinity for ochratoxin A and DON and relation of adsorption ability to physicochemical parameters. Journal of Food Protection, 61, 469-475.

Gong, Y.Y., Egal, S., Hounsa, A., Turner, P.C., Hall, A.J., Cardwell, K.F., Wild, C.P., 2003.

Food Res. Intern., 37, 985-1000.

Gong, Y.Y., Hounsa, A., Egal, S., Turner, P.C., Sutcliffe, A.E., Hall, A.J., Cardwell, K ., Wild, C.P., 2004. Environ. Health Perspect. 112, 1334-8.

Guan, J.G., Miao, Y.Q., Zang, Q.J., 2004. J. Biosci. Bioeng. 97, 219-226.

Gübitz, G., Schmid, M.G., Silviaeh, H., AboulEnein, H.Y., 2001. Critical Reviews in Analytical Chemistry. 31(2), 141-148.

Guerre, P., Eeckhoutte, C., Burgat, V., Galtier, P., 2000. Food Addit. Contam., 17, 1019-26.

Guntupalli, R., Hu, J., Lakshmanan, R., Huang, T., Barbaree, J., Chin, B., 2007. Biosensors and Bioelectronics. 22(7), 1474-9.

Halasz, A., Latsztity, R., Abonyi, T., Bata, A., 2009. Food Res. Int., 25, 284-98.

Hanif, N.Q., Muhammad, G., Siddique, M., Khanum, A., Ahmed, T., Gadahai, J.A., Kaukab, G., 2008. British Poultry Science. $49,632-642$.

Heineman, W.R., Jensen, W.B., 2006. Biosensors and Bioelectronics. 21(8), 1403-4.

Heurich, M., Abdul Kadir, M. K., Tothill, I. E., 2011. Sensors and Actuators B: Chemical 156: $162-8$.

Hideaki, Nakamura, Karube Isao. 2003. Analytical and bioanalytical chemistry 377 (3): 446-68.

Hofstetter, U., Schatzmayr, D., Starkl, V., Binder, E.M., Forat, M., 2006. A novel concept for simultaneous deactivation of various mycotoxins in piglets. BOKU symposium. Hoppenbrock, 02 November, 2006, pp. 80-4.

Horn, B.W., Wicklow, D.T., 1983. Can. J. Microbiol., 29, 1087-91.

Huff, W.E., L.F. Kubena, R.B. Harvey, and T.D. Phillips. 1992. Poult. Sci. 71:64-9. 
Ibraheem A., Campbell, R. E., 2010. Current Opinion in Chemical Biology. 14, 30-6.

Jares-Erijman E.A, Jovin, T.M., 2003. Nat. Biotechnol., 21, 1387-95.

Jaynes, W.F., Zartman, R.E., Hudnall, W.H., 2007. Applied Clay Science, 36, 197-205.

Kerppola, T.K., 2008a. Annu. Rev. Biophys. 37, 465-87.

Kerppola, T.K., 2008b. Methods Cell Biol. 85, 43170.

Kim, N., Park, I., Kim, D., 2007. Biosensors and Bioelectronics. 22(8), 1593-9.

Kirchner, P., Oberlander, J., Friedrich, P., Berger, J., Rysstad, G., Keusgen, M., Schoning, M.J., 2012. Sens. Actautors B: Chem.170, 60-66.

Kissell, L., Davidson, S., Hopkins, B.A., Smith, G.W., Whitlow, L.W., 2013. J. Anim. Physiol. Anim. Nutr. (Berl). 97(4), 694-700.

Kodama, Y., Wada, M., 2009. Plant Mol. Biol. 70, 211-217.

Kogan, G., Kocher, A., 2007. Livestock Science. 109, 161-5.

Koyun, A., Ahlatcioğlu, E., İpek, Y.K., 2012. Biosensors and Their Principles In: A Roadmap of Biomedical Engineers and Milestones, Prof. Sadik Kara (Ed.) InTech Europe publication, University Campus, Rijeka, Croatia.pp.115-42.

Lading, L., et. al., 2002. Comparing Biosensors", Sensors, proceedings of IEEE. 2, 229-232.

Lee, B.H., 2014. Fundamentals of Food Biotechnology. John Wiley \& Sons. pp. 544.

Li Dongqing., 2008. Encyclopedia of Microfluidics and Nanofluidics. Springer Science \& Business Media publisher. pp. 2226.

Li Songjun, Jagdish Singh, $\mathrm{He} \mathrm{Li}$, Ipsita A. Banerjee 2011. Biosensor Nanomaterials. John Wiley \& Sons. pp. 296.

Liedberg, B. Nylander C. and I. Lundstrom, Sens. Actuators, 1983, 4, 299-304.

Ligler, F.S., Taitt, C.R., 2008. Optical Biosensors, Second Edition: Today and Tomorrow. Elsevier B.V., Amsterdam, The Netherlands, pp712.

Liliana S. C., Jeyson G.P.V., 2011. Biosensors Applications in Agri-food Industry, Environmental Biosensors, Prof. Vernon Somerset (Ed.) InTech Europe publication, University Campus, Rijeka, Croatia.,pp.4364.

Lostis M. 1958. Ph.D. dissertation, Faculty of Science, University of Paris.

Lowe C.R., 1985. Biosensors, 1: 4.
Lud, S.Q., Nikolaides, M.G., Haase, I., Fischer, M., Bausch, A.R., 2006. Chem. Phys. Chem. 7 (2), 379-384.

Łukasz, Stepien., 2014. Critical Reviews in Microbiology. 40(2), 176-85.

Manbachi, A., Cobbold R.S.C., 2011. Ultrasound 19 (4), 187-196.

Marquardt, R.R., Frohlich, A.A., 1992. J. Anim. Sci., 70, 3968-88.

Marroquin-Cardona, A., Deng, Y., Taylor, J.F., Hallmark, C.T., Johnson, N., Phillips, T.D., 2009. Food Additives and Contaminants. 26, 733-43.

Maskow, T., Wolf, K., Kunze, W., Enders, S., Harms, H., 2012. Thermochim. Acta. 543, 273-80.

Meisner, H., Meisner, P., 1981. Arch. Biochem. Biophys. 208, 146-51.

Meissonnier, G.M., Raymond, I., Laffitte, J., Cossalter, A.M., Pinton, P., Benoit, E., Bertin, G., Galtier, P., Oswald, I.P., 2009. World Mycotoxin Journal. 2, 161-72.

Merrill, A.H,Jr., Sullards, M.C., Wang, E., Voss, K.A., Riley, R.T., 2001. Environ. Health Perspect. 109(Suppl. 2), 283-9.

Micheli, L., Grecco, R., Badea, M., Moscone, D. and Palleschi, G., 2005. Biosensors and Bioelectronics. 21, 588-596.

Milstein L. and Das P., 1977. Communications, IEEE Trans. 25(8), 841-847.

Mohanty, S.P., Kougianos E., 2006. IEEE Potentials. 25(2), $35-40$.

Moschini, M., Gallo, A., Piva, G., Masoero, F., 2008. Animal Feed Science and Technology. 147, 292-309.

Muhammad-Tahir, Z, Alocilja, E.C., 2003. Biosens. Bioelectron. 18, 813-19.

Mullett, W., Lai, E.P.C., Yeung, J.M., 1998. Analytical Biochemistry. 258,161-167.

Murphy, P. A., Suzanne, H., Cindy, L., Cory, B. M., 2006. Journal of Food Science. 71(5), 51-65.

Nageswara, S.B., Chopra, R.C., 2001. Small ruminent research, 41, 203-213.

Nakamura H., Hasegawa M., Arikawa Y., Nomura Y., Ikebukuro K. and I. Karube. 2003. Anal. Lett. 36, 1805-17.

Nakazato, M., Morozumi, S., Saito, K., Fujinuma, K., Nishima, T., Kasai, N., 1990. Applied and Envionmental Microbiology. 56, 1465-70.

Nasir, M.S., Jolley, M.E., 2002. Journal of Agricultural and Food Chemistry. 50, 311621.

Ngundi, M.M., Shriver-Lake, L.C., Moore, M. H., 
Lassman, M.E., Ligler, F.S., Taitt, C.R., 2005. Analytical Chemistry. 77, 148-154.

Ngundi, M.M., Shriver-Lake, L.C., Moore, M.H., Ligler, F.S., Taitt, C.R., 2006. Journal of Food Protection. 69, 3047-3051.

Niderkorn, V., Boudra, H., Morgavi, D.P., 2006. Journal of Applied Microbiology. 101, 84956.

Niderkorn, V., Boudra, H., Morgavi, D.P., 2008. World Mycotoxin Journal. 1, 463-67.

Niderkorn, V., Morgavi, D.P., Aboab, B., Lemaire, M., Boudra, H., 2009. Journal of Applied Microbiology. 106, 977-85.

Niderkorn, V., Morgavi, D.P., Pujos, E., Tissandier, A., Boudra, H., 2007. Food Additives and Contaminants. 24, 406-15.

Njobeh, P.B,, Dutton, M.F., Makun, A.H., 2010b. Mycotoxins and human health: Significance, prevention and control. In: Mishra $\mathrm{AK}$, Tiwari A, Mishra SB and Kobayashi H (Eds), Smart Biomolecules in Medicine. Research Signpost/Transworld Research Network. VBRI Press, India. pp. 132-77.

Paniel,

N.,Radoi,A.,Marty,J.L.,2010.Sensors10,9439 -9448 .

Parker, C., Lanyon, Y., Manning, M. Arrigan, D. W. M. and Tothill, I.E., 2009. Analytical Chemistry. 81, 5291-5298.

Parker, C., Tothill, I. E., 2009. Biosensors and Bioelectronics. 24, 2452-2457.

Patolsky, F.; Zayats, M.; Katz, E.; Willner, I. 1999. Analytical Chemistry. 71(15), 3171-3180.

Phillips, T.D., Afriyie-Gyawu, E., Williams, J., Huebner, H., Ankrah, N.-A., Ofori-Adjei, D., Jolly, P., Johnson, N., Taylor, J., MarroquinCardona, A., Xu, L., Tang, L., Wang, J.-S., 2008. Food Additives and Contaminants. 25, $134-145$.

Piatkevich, K.D., Verkhusha, V.V., 2010. Curr. Opin. Chem. Biol, 14, 23-9.

Picot, A., Barreau, C., Pinson-Gadais, L., Caron, D., Lannou, C., Richard-Forget, F., 2010. Critical Reviews in Microbiology. 36 (3). 221-31.

Piermarini, S., Micheli, L., Ammida, N.H.S., Palleschi, G., Moscone, D., 2007. Biosensors and Bioelectronics. 22, 1434-40.

Pohanka Miroslav, Skladal Petr., 2008. Journal of Applied Biomedicine. 6(2), 57-64.

Raj, H.G., Prasanna, H.R., Mage, P., Lotlikar, P.D., 1986. Cancer Lett. 33, 1-9.

Rastislav Monošík, Miroslav Stred'anský, Ernest Šturdík. 2012. Acta Chimica Slovaca. 5(1),
$109-120$

Richard, J.L., 2007. Inter. J. Food Microbiol., 119, 3-10.

Robert, R.G., Raymond, S.T., 1994. Appl. Environ. Microbiol., 60, 2864-68.

Rogers, K.R., 1995. Biosensors and Bioelectronics. 10, 533-41.

Sauerbrey G., 1959. Z. Phys., 155. p. 206.

Schnerr, H., Vogel, R.F., Niessen, L., 2002. Food and Agriculral Immunology. 14, 313-21.

Shephard, G.S., Marasas, W.F.O., Burger, H.M., Somdyala, N.I.M., Rheeder, J.P., Van der, Westhuizen, L., Gatyeni, P., Van Schalkwyk, D.J., 2007. Food Add. Contam. 24, 621-29.

Shuchen, H., Shu-Ling, H., Chiung-wen, H., PoChiao, L., Wu, Chun-Hsin., 2013. Journal of Analytical Methods in Chemistry. Vol. 2013, Article ID 687265. pp. 5.

Smith, T.K., MacDonald, E.J., Haladi, S., 2008. Current concepts in feed-borne mycotoxins and the potential for dietary prevention of mycotoxicoses. Engormix.com.

Stockbridge C.D., 1996. Vac. Microbalance Tech., 5. p. 193.

Su T, Zhang Z and Luo Q., 2012. Biosens. Bioelectron., 31(1), 292-8.

Tangni, E.K., 2003. Occurrence of mycotoxins in beer, exposure assessment for consumers and development of biological detoxification options for the control of ochratoxin A during brewing. Ph.D. Thesis. Belgium: Universite Catholique De Louvain, 2003.

Tangni, E.K., De Rouck, G., Potel, A., De Meeus, L., Aerts, G., Larondelle, Y., 2005. Towards mycotoxin control in brewing: Adfimax ${ }^{\circledR}$ as a novel promising solution to overcome this challenge. 30Th, European Brewery Convention; 2005; Prague. pp.161.

Thompson, V.S., Maragos, C.M., 1996. Journal of Agricultural and Food Chemistry. 44, 104146.

Thrasher, J.D., Crawley, S., 2009. Toxicol. Ind. Health. 25(9-10), 583-615.

Tothill, I.E., 2011. World Mycotoxin Journal. 4 (4), 361-374.

Tothill, I.E., Turner, A.P.F., 2003. Biosensors, In: Encyclopaedia of Food Sciences and Nutrition (Sec. Ed.), Benjamin C., Luiz T., Paul F., Editors, Academic Press, pp 489-499.

Trckova, M., Matlova, L., Dvorska, L., Pavlik, I., 2004. Veterinary medicine. 49, 389-399.

Tsai, W.C., Hsieh, C.K., 2007. Analytical Letters, 40(10), 1979-91. 
Tudos, A. J., Lucas van den Bos, E. R., Stigter, E.C.A., 2003. Journal of Agriculture and Food Chemistry. 51, 5843-5848.

Turner A.P.F., 1989. Sensors Actuators, 17:433450.

Turner, P.C., Moore, S.E., Hall, A.J., Prentice, A.M., Wild, C.P., 2003. Health Perspec. 111, 217-20.

Turner, A., Wilson, G., Kaube, I., 1987. Biosensors: Fundamentals and Applications. Oxford, UK: Oxford University Press. pp. 770.

Upadhaya, S.D., Park, M.A., Jong, K.H., 2010. Asin-Aust. J. Anim. Sci., 23(9), 1250-60.

URL1:

https://en.wikipedia.org/wiki/Biosensor\#cite_ note-1

Valach, M., Katrlik, J., Sturdik, E. \& Gemeiner, P., 2009. Sensors and Actuators B: Chemical. 138(2), 581-86.

Valeur, Bernard., 2001. Molecular Fluorescence: Principles and Applications. WileyVCHVerlag GmbH. pp. 381.

Välimaa, A.L., Kivistö, A.T., Leskinen, P.I., Karp, M.T., 2010. J. Microbiol. Methods. 80(1), 448.

Walton P. W., O'Flaherty M. R., Butler M. E., Compton P., 1993. Biosensors and Bioelectronics. 8(9-10), 401-7.

Wang, P., Afriyie-Gyawu, E., Tang, Y., Johnson, N., Xu, L., Tang, L., Huebner, H., Ankrah,
N.-A., Ofori-Adjei, D., Ellis, R.W., Jolly, P., Williams, J., Wang, J.-S., Phillips, T.D., 2008. Food Additives and Contaminants. 25, 622-34.

Weckbach, L.S., Marth, E.H.,1977. Mycopathologia. 62, 39-45.

Wicklow, D.T., Hesseltine,C.W., Shotwell, O.L., Adams, G.L.,1980. Phytopathology. 70, 761764.

Wicklow, D.T., Horn, B.W., Shotwell, O.L., Hesseltine, C.W., Caldwell, R.W., 1988. Phytopathology. 78, 68-74.

Wood R.W., 1902. Philos. Mag., 4, 396-402.

Wood R.W., 1912, Philos. Mag., 23, 310-317.

Xie, B., Ramanathan, K., Danielsson, B. 1999. Adv. Biochem. Eng. 64, 1-33.

$\mathrm{Xu}$ Qinglong, Kimihiko Sato and Shotaro Yamaguchi., 2014. Steamed bun quality improving agent and use thereof Pub. No: US 20140037790 A1.pp.1-12.

Xu, Q., Sato, K., Shotaro, Y., 2014. Steamed bun quality improving agent and use thereof Pub. No: US 20140037790 A1.pp.1-12.

Yiannikouris, A., Andre, G., Buleon, A., Jeminet, G., Canet, I., Francois, J., Bertin, G., Jouany, J.P., 2004. Biomacromolecules, 5, 2176-85.

Zinedine, A., Soriano, J. M., Moltó, J.C., Mañes, J., 2007. Food and Chemical Toxicology. 45, 118.

\section{How to cite this article:}

Shrvan Kumar, Asha Sinha, Shweta Meshram, Mukesh Kumar Singh, Vimla Singh and K.S. Hooda. 2018. Myco-toxins Monitoring Device and their Management Strategies through Detoxifying Agents in Feed. Int.J.Curr.Microbiol.App.Sci. 7(01): 3410-3426.

doi: https://doi.org/10.20546/ijcmas.2018.701.403 